\title{
Analytic calculation of the electric field of a coherent THz pulse
}

\author{
Markus Schwarz, Philipp Basler, Matthias v. Borstel, and Anke-Susanne Müller \\ Karlsruhe Institute of Technology, Kaiserstraße 12, 76131 Karlsruhe, Germany
}

(Received 15 February 2014; published 12 May 2014)

\begin{abstract}
Accelerators can serve as sources for intense THz radiation by producing sub-ps long electron bunches, which generate synchrotron radiation coherently in the THz regime. In this paper, we present an analytic method to calculate the electric field pulse emitted by an electron bunch with arbitrary bunch shape. Our method is applied to a Gaussian bunch as well as a complex bunch profile resulting from a nonlinear beam dynamics simulation.
\end{abstract}

DOI: 10.1103/PhysRevSTAB.17.050701

PACS numbers: 41.60.Ap, 42.25.Kb, 02.60.Ed

\section{INTRODUCTION}

Coherent radiation is produced whenever the emitted wavelength is comparable or larger than the source size. Its intensity increases with the number of emitters squared, as opposed to the incoherent radiation, which increases only linearly. Since we take an electron bunch as a source, consisting of several hundred million electrons, coherent radiation from electron bunches is enhanced over incoherent radiation by this factor. For coherent radiation to be in the $\mathrm{THz}$ regime, the electron bunch must be shorter than 1 ps. Progress in accelerator science has enabled the creation of coherent $\mathrm{THz}$ radiation in storage rings $[1,2]$. However, further decreasing the bunch size, and, therefore, reaching frequencies above $1 \mathrm{THz}$, is limited by instabilities. These instabilities are not present in linear accelerators. The linear accelerator FLUTE [3], which is currently under construction at Karlsruhe Institute of Technology, allows the production of bunches with bunch lengths ranging from 100 fs to a few fs and charges from a few $\mathrm{nC}$ to $\mathrm{pC}$, respectively.

Because the coherence condition depends on both the bunch shape and the frequency, the spectrum of coherent radiation contains information on the longitudinal bunch profile. Conversely, due to the large amount of electrons, even small substructures in a bunch can lead to a significant production of coherent radiation. Thus, coherent radiation offers a tool for the noninvasive study of bunch profiles and beam dynamics [4].

Besides the spectrum of the emitted $\mathrm{THz}$ radiation, the temporal profile as well as the peak value of the corresponding electric field pulse are important [5]. To design an accelerator that optimizes these parameters requires the calculation of the electric field pulse from the bunch profile.

\footnotetext{
*Markus.Schwarz@kit.edu

Published by the American Physical Society under the terms of the Creative Commons Attribution 3.0 License. Further distribution of this work must maintain attribution to the author $(s)$ and the published article's title, journal citation, and DOI.
}

For this, one needs to sum the electric fields at a given frequency emitted by each electron and then integrate over all frequencies. Since the number of electrons in a bunch is large, the sum can be replaced by an integral over the bunch profile. The resulting double integral could, in principle, be performed numerically for an arbitrary bunch profile. However, it turns out that the numerical integration converges poorly and we resort to analytic methods in this paper. For Gaussian profiles the pulse shape of a coherent $\mathrm{THz}$ pulse has been calculated analytically in [6,7]. In this paper, we present an analytic method that applies to arbitrary bunch shapes. The method is based on first doing a cubic spline interpolation of an arbitrary bunch profile. Subsequently, the integrals over the bunch profile and frequency are computed analytically and expressed in terms of the coefficients of the spline interpolation.

The paper is organized as follows: In Sec. II we derive how the electric field pulse can be calculated from a spectral integral. The field is then calculated analytically for a Gaussian electron bunch profile in Sec. II A. Next, in Sec. II B, we derive our main result: an expression for the electric field of an arbitrary bunch shape based on a cubic spline interpolation. The two methods are compared in Sec. III A for a Gaussian bunch. Finally, we employ the spline interpolation in Sec. III B to compute the electric field of a simulated complex bunch profile. Section IV discusses the results.

\section{DERIVATION}

The electric field pulse coherently emitted by an electron bunch is obtained by summing over the individual fields of all electrons [8,9]. Here, we assume that the field of a single electron, as detected by an observer at distance $R$ and time $t$, is given by a plane wave propagating in the positive $z$-direction [7]

$$
E_{\omega}(R, t)=\operatorname{Re}\left(E_{0}(\omega) \mathrm{e}^{-\mathrm{i} \omega(t-R / c)-\mathrm{i} \phi}\right),
$$

where $c$ denotes the speed of light. The details of the radiation mechanism result in a frequency dependence of 
the spectral amplitude $E_{0}(\omega)$. In the following, the phase $\phi$ is assumed to be constant for all electrons. For two electrons, the field is the superposition of two plane waves, but the wave of the second electron has an additional phase factor of $i \omega \Delta \tau$, which takes the relative displacement $\Delta \tau$ of the two electrons into account. Summing over all $N$ electrons we obtain

$$
\begin{aligned}
E_{\omega}(R, t) & =E_{0}(\omega) \operatorname{Re}\left(\mathrm{e}^{-\mathrm{i} \omega(t-R / c)-\mathrm{i} \phi} \sum_{i=1}^{N} \mathrm{e}^{\mathrm{i} \omega \Delta \tau_{i}}\right) \\
& \rightarrow N E_{0}(\omega) \operatorname{Re}\left(\mathrm{e}^{-\mathrm{i} \omega(t-R / c)-\mathrm{i} \phi} \int \mathrm{e}^{\mathrm{i} \omega \tau} \varrho(\tau) \mathrm{d} \tau\right) .
\end{aligned}
$$

In the second line, we took the continuum limit and replaced the sum over all electrons by an integral over the normalized charge distribution $\varrho(\tau)$. Notice that the integral is the Fourier transform $\mathcal{F}$ of the charge distribution and $\varrho(\omega) \equiv \mathcal{F}[\varrho(t)]$ in the following. Finally, we integrate over the whole spectrum and the result reads

$$
E(R, t)=N \operatorname{Re}\left(\mathrm{e}^{-\mathrm{i} \phi} \int_{0}^{\infty} E_{0}(\omega) \varrho(\omega) \mathrm{e}^{-\mathrm{i} \omega(t-R / c)} \mathrm{d} \omega\right) .
$$

In the following, we set $R$ to zero in Eq. (3) for convenience, as it is merely a constant shift in observation time $t$. To solve Eq. (3) we must first calculate the Fourier transform $Q(\omega)$ and then solve the spectral integral.

Our main focus is on coherent synchrotron radiation (CSR) as the radiation mechanism. As the spectral amplitude $E_{0}(\omega)$ we take the angle integrated synchrotron spectrum $[10,11]$

$$
\begin{aligned}
E_{0}(\omega) & \equiv \sqrt{\int|\vec{E}(\omega)|^{2} \mathrm{~d} \Omega}=\sqrt{\int \frac{1}{2 \epsilon_{0} c} \frac{\mathrm{d} I}{\mathrm{~d} \omega \mathrm{d} \Omega} \mathrm{d} \Omega} \\
& \simeq \frac{e \sqrt{\gamma}}{\sqrt{4 \pi} \epsilon_{0} c R} \frac{27^{1 / 4}}{4^{1 / 3}} \sqrt{\Gamma(5 / 3)}\left(\frac{\omega}{\omega_{c}}\right)^{1 / 6} \\
& =\frac{3^{7 / 12} e}{\sqrt{8 \pi} \epsilon_{0} c^{7 / 6} R} \sqrt{\Gamma(5 / 3)}(\omega \rho)^{1 / 6},
\end{aligned}
$$

with $e$ and $\epsilon_{0}$ denoting the electric charge and vacuum permittivity, respectively. In the second line, we used the low frequency approximation, valid for $\omega \ll \omega_{c}$. Finally, we substituted the definition of the critical frequency $\omega_{c} \equiv$ $3 \gamma^{3} c / 2 \rho$ in terms of the bending radius $\rho$. Notice that the final result is independent of the energy $\gamma$. Also notice that the use of Eq. (4) in the spectral integral of Eq. (3) would lead to a UV divergence, if it were not for the highfrequency cutoff provided by the Fourier transformed charge distribution $\rho(\omega)$. The latter goes to zero for frequencies larger than the inverse bunch length $\sigma_{b}$. The use of the low frequency spectrum is, therefore, justified as long as $1 \ll \sigma_{b} \omega_{c}$. If this condition is not satisfied, i.e., for very short bunches, the upper limit of integration in Eq. (3) must be replaced by $\omega_{c}$. The cutoff in this case is provided by $\omega_{c}$ because the synchrotron spectrum decays as $\sqrt{\omega / \omega_{c}} \exp \left(-\omega / \omega_{c}\right)$ for frequencies larger than $\omega_{c}$ [10]. For the remainder of the article, we assume $1 \ll \sigma_{b} \omega_{c}$ and use Eq. (4).

The main problem is then to compute

$$
\varepsilon(t) \equiv \int_{0}^{\infty} \omega^{1 / 6} \varrho(\omega) \mathrm{e}^{-\mathrm{i} \omega t} \mathrm{~d} \omega,
$$

for a given charge distribution. The coherent electric field pulse of a bunch with charge $Q$ is then given by

$$
\begin{aligned}
E(t)= & \frac{3^{7 / 12} \sqrt{\Gamma(5 / 3)} Q \rho^{1 / 6}}{\sqrt{8 \pi} \epsilon_{0} c^{7 / 6} R} \\
& \times\{\cos \phi \operatorname{Re}[\varepsilon(t)]+\sin \phi \operatorname{Im}[\varepsilon(t)]\}
\end{aligned}
$$

\section{A. Gaussian bunch}

In this section we consider a Gaussian bunch of width $\sigma_{b}$, that is, the profile is given by

$$
\varrho(t)=\frac{1}{\sqrt{2 \pi} \sigma_{b}} \mathrm{e}^{-t^{2} / 2 \sigma_{b}^{2}}
$$

The Fourier transform is well known and reads explicitly

$$
\varrho(\omega)=\mathrm{e}^{-\omega^{2} \sigma_{b}^{2} / 2} .
$$

Inserting Eq. (7) into Eq. (5) and splitting into real and imaginary parts leads to

$$
\begin{aligned}
\operatorname{Re} \varepsilon(t) & =\int_{0}^{\infty} \omega^{1 / 6} \mathrm{e}^{-\omega^{2} \sigma_{b}^{2} / 2} \cos (\omega t) \mathrm{d} \omega \\
& =\frac{2^{-5 / 12} \Gamma\left(\frac{7}{12}\right)}{\sigma_{b}^{7 / 6}}{ }_{1} F_{1}\left(\frac{7}{12}, \frac{1}{2} ;-\frac{t^{2}}{2 \sigma_{b}^{2}}\right), \\
-\operatorname{Im} \varepsilon(t) & =\int_{0}^{\infty} \omega^{1 / 6} \mathrm{e}^{-\omega^{2} \sigma_{b}^{2} / 2} \sin (\omega t) \mathrm{d} \omega \\
& =\frac{2^{1 / 12} \Gamma\left(\frac{13}{12}\right)}{\sigma_{b}^{7 / 6}} \frac{t}{\sigma_{b}}{ }_{1} F_{1}\left(\frac{13}{12}, \frac{3}{2} ;-\frac{t^{2}}{2 \sigma_{b}^{2}}\right) .
\end{aligned}
$$

These integrals were solved using Eqs. (3.952,7-8) of [12] together with the property $\exp (x)_{1} F_{1}(b-a ; b ;-x)=$ ${ }_{1} F_{1}(a ; b ; x)$ and ${ }_{1} F_{1}(a, b ; x)$ denotes Kummer's confluent hypergeometric function. With $\tau \equiv t / \sigma_{b}$ denoting time in units of bunch length, the electric field pulse emitted by a Gaussian bunch reads 


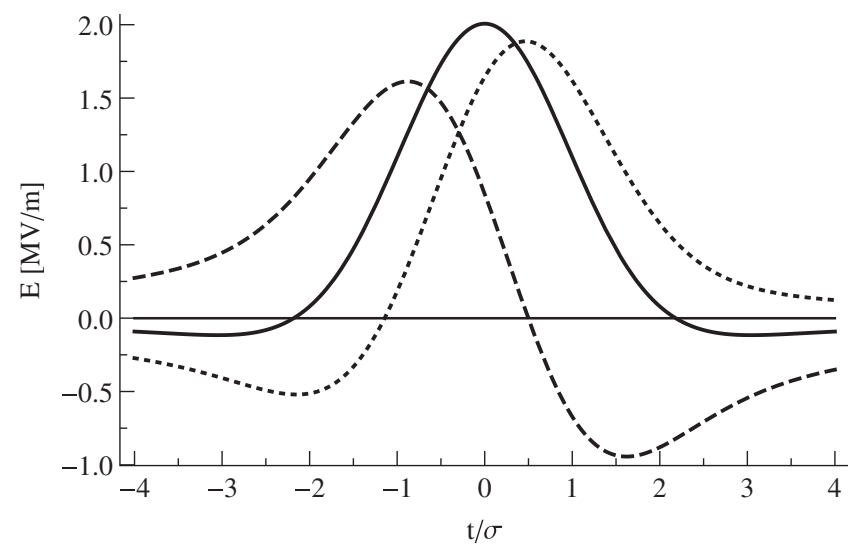

FIG. 1. Time dependence of the electric field $E$, coherently emitted by a Gaussian bunch with length $\sigma$ according to Eq. (9). The parameters are $Q=100 \mathrm{pC}, R=1 \mathrm{~m}, \rho=1.1 \mathrm{~m}$, and $\sigma_{b}=50$ fs. For $\phi=0$ (continuous) we get a symmetric socalled half-cycle pulse whereas $\phi=65^{\circ}$ (dashed) and $\phi=325^{\circ}$ (dotted) yield single-cycle pulses with minima at $t>0$ and $t<0$, respectively.

$$
\begin{aligned}
E(t)= & \frac{3^{7 / 12} \sqrt{\Gamma(5 / 3)} Q \rho^{1 / 6}}{\sqrt{4 \pi} \epsilon_{0} 2^{11 / 12}\left(\sigma_{b} c\right)^{7 / 6} R} \\
& \times\left\{\cos \phi \Gamma\left(\frac{7}{12}\right){ }_{1} F_{1}\left(\frac{7}{12}, \frac{1}{2} ;-\frac{\tau^{2}}{2}\right)\right. \\
& \left.-\sin \phi \sqrt{2} \Gamma\left(\frac{13}{12}\right) \tau_{1} F_{1}\left(\frac{13}{12}, \frac{3}{2} ;-\frac{\tau^{2}}{2}\right)\right\} .
\end{aligned}
$$

Figure 1 shows a plot of the electric field for a Gaussian bunch with $\sigma_{b}=50$ fs for different values of the phase $\phi$. For $\phi=0$ we get a so-called half-cycle pulse which is symmetric and centered at $t=0$, corresponding to the center of the charge distribution. Notice that the pulse shape resembles the underlying Gaussian charge profile. For $0<\phi<180^{\circ}$ a minimum develops at $t>0$ (see dashed curve in Fig. 1), leading to a single-cycle pulse, which is antisymmetric at $\phi=90^{\circ}$. The pulse shape for $\phi=180^{\circ}$ is the negative of the one for $\phi=0$. Phases with $180^{\circ}<\phi<$ $360^{\circ}$ have a minimum at $t<0$ (see dotted curve in Fig. 1). However, the integral over all time is zero in all cases [13]. This implies that the larger the minimum, the smaller the maximum field becomes, as can be seen in Fig. 1.

An arbitrary bunch profile can be written as a sum of Gaussians. The electric field, being linear in $\rho(\omega)$, is then a superposition of fields given by Eq. (9). Using a multiGaussian fit is possible, but becomes tedious in practice.

\section{B. Arbitrary bunch shape}

We proceed in three steps to analytically compute Eq. (5) for an arbitrary bunch shape. The first step is to use a spline interpolation for the bunch profile. Second, we compute the Fourier-transform $\varrho(\omega)$ of the interpolated profile.
The integral in Eq. (5) can then be calculated analytically in the third step.

\section{Spline interpolation}

We assume that the bunch profile is given at $N+1$ discrete, not necessarily equidistant, points $\left(t_{i}, \varrho_{i}\right)$, with $i=0 \ldots N$. The spline interpolation connects these points by cubic polynomials, such that the resulting curve is continuous up to the second derivative. The cubic polynomial $\varrho_{i}(t)$ that interpolates between the points $\left(t_{i-1}, \varrho_{i-1}\right)$ and $\left(t_{i}, Q_{i}\right)$ is given by

$$
\begin{aligned}
\varrho_{i}(t)= & \left(c_{i, 3} t^{3}+c_{i, 2} t^{2}+c_{i, 1} t+c_{i, 0}\right) \\
& \times\left[\Theta\left(\Delta t_{i-1}\right)-\Theta\left(\Delta t_{i}\right)\right], \quad i=1, \ldots, N,
\end{aligned}
$$

where $\Delta t_{i} \equiv t-t_{i}$. The Heaviside functions $\Theta(t)$ ensure that the polynomial "lives" only between $t_{i-1}$ and $t_{i}$ and is zero outside this interval. For each of the $N$ intervals $\left[t_{i-1}, t_{i}\right]$ the coefficients $c_{3}$ to $c_{0}$ need to be determined such that

$$
\begin{aligned}
\varrho_{i}\left(t_{i}\right) & =\varrho_{i}, \quad \varrho_{i-1}^{\prime}\left(t_{i-1}\right)=\varrho_{i}^{\prime}\left(t_{i-1}\right), \\
\varrho_{i}\left(t_{i-1}\right) & =\varrho_{i-1}, \quad \varrho_{i-1}^{\prime \prime}\left(t_{i-1}\right)=\varrho_{i}^{\prime \prime}\left(t_{i-1}\right) .
\end{aligned}
$$

In terms of the second derivatives at the data points $p_{i} \equiv \varrho_{i}^{\prime \prime}\left(t_{i}\right)$, the coefficients then read [14]

$$
\begin{gathered}
c_{3, i}=\frac{p_{i}-p_{i-1}}{6\left(t_{i}-t_{i-1}\right)}, \\
c_{2, i}=\frac{t_{i} p_{i-1}-t_{i-1} p_{i}}{2\left(t_{i}-t_{i-1}\right)}, \\
c_{1, i}=\frac{\varrho_{i}-\varrho_{i-1}}{t_{i}-t_{i-1}}-c_{2, i} \frac{t_{i}^{2}-t_{i-1}^{2}}{t_{i}-t_{i-1}}-c_{3, i} \frac{t_{i}^{3}-t_{i-1}^{3}}{t_{i}-t_{i-1}}, \\
c_{0, i}=\varrho_{i}-c_{3, i} t_{i}^{3}-c_{2, i} t_{i}^{2}-c_{1, i} t_{i},
\end{gathered}
$$

with $i=1, \ldots, N$. Of course, Eq. (11c) can be simplified, but it will be more convenient in this form later. The $p_{i}$ themselves can be computed very efficiently numerically [15], given the free boundary values $p_{0}$ and $p_{N}$. Here, we use the boundary values for natural splines, that is

$$
p_{0}=p_{N}=0 .
$$

\section{Fourier transform}

With the coefficients in Eq. (10) expressed in terms of the data points, we can now proceed to compute the Fourier transform $\varrho_{i}(\omega)$. From Eq. (10) we see that we have to compute integrals of the form

$$
\int c_{n, i} t^{n}\left[\Theta\left(\Delta t_{i-1}\right)-\Theta\left(\Delta t_{i}\right)\right] \mathrm{e}^{\mathrm{i} \omega t} \mathrm{~d} t
$$


with $n=0, \ldots, 3$. For $n=0$ we have

$$
\begin{aligned}
& \int c_{0, i}\left[\Theta\left(\Delta t_{i-1}\right)-\Theta\left(\Delta t_{i}\right)\right] \mathrm{e}^{\mathrm{i} \omega t} \mathrm{~d} t \\
& =c_{0, i}\left(\mathrm{e}^{\mathrm{i} \omega t_{i-1}}-\mathrm{e}^{\mathrm{i} \omega t_{i}}\right) \int \Theta(t) \mathrm{e}^{\mathrm{i} \omega t} \mathrm{~d} t \\
& =c_{0, i}\left(\mathrm{e}^{\mathrm{i} \omega t_{i-1}}-\mathrm{e}^{\mathrm{i} \omega t_{i}}\right)\left[\frac{\mathrm{i}}{\omega}+\pi \delta(\omega)\right] \\
& =c_{0, i}\left(\mathrm{e}^{\mathrm{i} \omega t_{i-1}}-\mathrm{e}^{\mathrm{i} \omega t_{i}}\right) \frac{\mathrm{i}}{\omega},
\end{aligned}
$$

where we used Eq. (17.23,6) of [12] for the Fourier transform of the Heaviside function $\Theta(t)$ and $\delta(\omega)$ denotes the Dirac $\delta$-function. Results for other powers of $n$ can be obtained by applying

$$
\mathcal{F}\left[t^{n} f(t)\right]=(-\mathrm{i})^{n} f^{(n)}(\omega),
$$

to Eq. (13), where $f^{(n)}$ denotes the $n$th derivative of a function $f$. The contribution from the $\delta$-function vanishes because the exponential functions cancel for $\omega=0$. The same is true for the higher order derivatives of the $\delta$-function appearing for $n>0$ [14]. We then have

$$
\begin{aligned}
\varrho(\omega)= & \sum_{i=1}^{N}\left\{\frac{1}{\omega^{4}}\left[6 c_{3, i}\left(\mathrm{e}^{\mathrm{i} \omega t_{i-1}}-\mathrm{e}^{\mathrm{i} \omega t_{i}}\right)\right]-\frac{\mathrm{i}}{\omega^{3}}\left[6 c_{3, i}\left(t_{i-1} \mathrm{e}^{\mathrm{i} \omega t_{i-1}}-t_{i} \mathrm{e}^{\mathrm{i} \omega t_{i}}\right)+2 c_{2, i}\left(\mathrm{e}^{\mathrm{i} \omega t_{i-1}}-\mathrm{e}^{\mathrm{i} \omega t_{i}}\right)\right]\right. \\
& -\frac{1}{\omega^{2}}\left[3 c_{3, i}\left(t_{i-1}^{2} \mathrm{e}^{\mathrm{i} \omega t_{i-1}}-t_{i}^{2} \mathrm{e}^{\mathrm{i} \omega t_{i}}\right)+2 c_{2, i}\left(t_{i-1} \mathrm{e}^{\mathrm{i} \omega t_{i-1}}-t_{i} \mathrm{e}^{\mathrm{i} \omega t_{i}}\right)+c_{1, i}\left(\mathrm{e}^{\mathrm{i} \omega t_{i-1}}-\mathrm{e}^{\mathrm{i} \omega t_{i}}\right)\right] \\
& \left.+\frac{\mathrm{i}}{\omega}\left[c_{3, i}\left(t_{i-1}^{3} \mathrm{e}^{\mathrm{i} \omega t_{i-1}}-t_{i}^{3} \mathrm{e}^{\mathrm{i} \omega t_{i}}\right)+c_{2, i}\left(t_{i-1}^{2} \mathrm{e}^{\mathrm{i} \omega t_{i-1}}-t_{i}^{2} \mathrm{e}^{\mathrm{i} \omega t_{i}}\right)+c_{1, i}\left(t_{i-1} \mathrm{e}^{\mathrm{i} \omega t_{i-1}}-t_{i} \mathrm{e}^{\mathrm{i} \omega t_{i}}\right)+c_{0, i}\left(\mathrm{e}^{\mathrm{i} \omega t_{i-1}}-\mathrm{e}^{\mathrm{i} \omega t_{i}}\right)\right]\right\} .
\end{aligned}
$$

The sums with odd powers of $\omega$ can further be simplified by inserting the values of the $c_{n, i}$ from Eqs. (11) [14]. For the sum proportional to $1 / \omega^{3}$ we obtain

$$
\begin{aligned}
& \sum_{i=1}^{N}\left(6 c_{3, i} t_{i-1}+2 c_{2, i}\right) \mathrm{e}^{\mathrm{i} \omega t_{i-1}}-\left(6 c_{3, i} t_{i}+2 c_{2, i}\right) \mathrm{e}^{\mathrm{i} \omega t_{i}} \\
& =\sum_{i=1}^{N} p_{i-1} \mathrm{e}^{\mathrm{i} \omega t_{i-1}}-p_{i} \mathrm{e}^{\mathrm{i} \omega t_{i}}=p_{0} \mathrm{e}^{\mathrm{i} \omega t_{0}}-p_{N} \mathrm{e}^{\mathrm{i} \omega t_{N}}=0 .
\end{aligned}
$$

In the last step, we used the boundary condition for natural splines, Eq. (12). Similarly, the sum proportional to $1 / \omega$ reduces to

$$
\sum_{i=1}^{N} \varrho_{i-1} \mathrm{e}^{\mathrm{i} \omega t_{i-1}}-\varrho_{i} \mathrm{e}^{\mathrm{i} \omega t_{i}}=\left(\varrho_{0} \mathrm{e}^{\mathrm{i} \omega t_{0}}-\varrho_{N} \mathrm{e}^{\mathrm{i} \omega t_{N}}\right)=0
$$

Here, we assumed that the charge distribution approaches zero at its endpoints. The Fourier transform in Eq. (14) then simplifies to

$$
\begin{aligned}
\varrho(\omega)= & \sum_{i=1}^{N}\left\{\frac{1}{\omega^{4}}\left[6 c_{3, i}\left(\mathrm{e}^{\mathrm{i} \omega t_{i-1}}-\mathrm{e}^{\mathrm{i} \omega t_{i}}\right)\right]\right. \\
& +\frac{1}{\omega^{2}}\left[\left(3 c_{3, i} t_{i}^{2}+2 c_{2, i} t_{i}+c_{1, i}\right) \mathrm{e}^{\mathrm{i} \omega t_{i}}\right. \\
& \left.\left.-\left(3 c_{3, i} t_{i-1}^{2}+2 c_{2, i} t_{i-1}+c_{1, i}\right) \mathrm{e}^{\mathrm{i} \omega t_{i-1}}\right]\right\} .
\end{aligned}
$$

\section{Electric field}

Inserting Eq. (15) into Eq. (5) leads to a sum of integrals of the form

$$
\varepsilon(t) \propto \sum_{i=1}^{N} c_{n, i} \int_{0}^{\infty} \omega^{-\nu} \mathrm{e}^{-\mathrm{i} \omega \Delta t_{i}} \mathrm{~d} \omega
$$

with $\nu$ either $11 / 6$ or $23 / 6$. Splitting into real and imaginary parts leads to the sine- and cosine transformations [see Eqs. $(17.33,2)$ and $(17.34,1)$ of [12]]

$$
\int_{0}^{\infty} \frac{\cos (\omega \Delta t)}{\omega^{\nu}} \mathrm{d} \omega=\sin \left(\frac{\pi}{2} \nu\right) \Gamma(1-\nu) \Delta t^{\nu-1}, \quad 0<\nu<1,
$$

$$
\int_{0}^{\infty} \frac{\sin (\omega \Delta t)}{\omega^{\nu}} \mathrm{d} \omega=\cos \left(\frac{\pi}{2} \nu\right) \Gamma(1-\nu) \Delta t^{\nu-1}, \quad 0<\nu<2 .
$$

There are, however, two problems in directly applying Eqs. (17a), (17b) to Eq. (16). First, Eqs. (17a), (17b) are true only for $\Delta t>0$, whereas $\Delta t$ can have any sign since $t$ can be either less or greater than any of the $t_{i}$. Second, $\nu=11 / 6>1$ preventing the use of Eqs. (17a) and for $\nu=23 / 6>2$ the sine transform cannot be applied. The solution to the first problem is to rewrite

$$
\begin{aligned}
& \cos (\omega \Delta t)=\cos (\omega|\Delta t|), \\
& \sin (\omega \Delta t)=\operatorname{sgn}(\Delta t) \sin (\omega|\Delta t|),
\end{aligned}
$$

and use the right-hand side instead [16].

The second problem requires more effort. Its root is the IR behavior of the integral. Using a Taylor expansion around $\omega=0$ we find 


$$
\begin{aligned}
\int \omega^{-\nu} \cos (\omega \Delta t) \mathrm{d} \omega & \simeq \int \omega^{-\nu} 1 \mathrm{~d} \omega \propto \omega^{1-\nu}, \\
\int \omega^{-\nu} \sin (\omega \Delta t) \mathrm{d} \omega & \simeq \int \omega^{-\nu} \omega \mathrm{d} \omega \propto \omega^{2-\nu} .
\end{aligned}
$$

The former integral converges only for $0<\nu<1$, while the latter converges for $0<\nu<2$, giving rise to the conditions in Eqs. (17a), (17b). Now, the crucial point is that the integrals in Eq. (16) appear not in an isolated form, but in a sum together with the coefficients of Eqs. (11). For example, for the sine transform with $\nu=23 / 6$ we find

$$
\begin{aligned}
& \omega^{-23 / 6} \sum_{i=1}^{N} 6 c_{3, i}\left[\sin \left(\omega t_{i-1}\right)-\sin \left(\omega t_{i}\right)\right] \\
& \simeq \omega^{-23 / 6} \sum_{i=1}^{N} 6 c_{3, i} \omega\left[t_{i-1}-t_{i}\right] \\
& =\omega^{-17 / 6} \sum_{i=1}^{N} 6 \frac{p_{i}-p_{i-1}}{6\left(t_{i}-t_{i-1}\right)}\left(t_{i-1}-t_{i}\right) \\
& =\omega^{-17 / 6} \sum_{i=1}^{N} p_{i}-p_{i-1}=0 .
\end{aligned}
$$

In the first step, we expanded the sin-functions to first order around $\omega=0$, then inserted Eq. (11a) for $c_{3, i}$ in the second step, and finally used the condition for natural splines, Eq. (12). The diverging parts of these two integrals, thus, cancel. Expanding the sin-function up to third order yields $\omega^{-5 / 6}$, which integrates to $\omega^{+1 / 6}$ and does not diverge at $\omega=0$. It turns out that the other divergent parts of the integrals in the sum in Eq. (16) cancel as well [16]. We, thus, can use the following

$$
\begin{aligned}
\int_{0}^{\infty} \omega^{-\nu} \mathrm{e}^{-\mathrm{i} \omega \Delta t_{i}}= & \Gamma(1-\nu)\left[\sin \left(\frac{\pi \nu}{2}\right)\right. \\
& \left.-\operatorname{isgn}\left(\Delta t_{i}\right) \cos \left(\frac{\pi \nu}{2}\right)\right]\left|\Delta t_{i}\right|^{\nu-1} .
\end{aligned}
$$

Using Eq. (18) then yields our main result

$$
\begin{aligned}
\varepsilon(t)= & \Gamma\left(\frac{-5}{6}\right) \sum_{i=1}^{N}-\frac{216}{187} c_{3, i}\left\{\left|\Delta t_{i}\right|^{17 / 6}\left[\sin \left(\frac{23 \pi}{12}\right)-\operatorname{isgn}\left(\Delta t_{i}\right) \cos \left(\frac{23 \pi}{12}\right)\right]\right. \\
& \left.-\left|\Delta t_{i-1}\right|^{17 / 6}\left[\sin \left(\frac{23 \pi}{12}\right)-\operatorname{isgn}\left(\Delta t_{i-1}\right) \cos \left(\frac{23 \pi}{12}\right)\right]\right\} \\
& +\left(3 c_{3, i} t_{i}^{2}+2 c_{2, i} t_{i}+c_{1, i}\right)\left|\Delta t_{i}\right|^{5 / 6}\left[\sin \left(\frac{11 \pi}{12}\right)-\operatorname{isgn}\left(\Delta t_{i}\right) \cos \left(\frac{11 \pi}{12}\right)\right] \\
& -\left(3 c_{3, i} t_{i-1}^{2}+2 c_{2, i} t_{i-1}+c_{1, i}\right)\left|\Delta t_{i-1}\right|^{5 / 6}\left[\sin \left(\frac{11 \pi}{12}\right)-\operatorname{isgn}\left(\Delta t_{i-1}\right) \cos \left(\frac{11 \pi}{12}\right)\right] .
\end{aligned}
$$

This final result for $\varepsilon(t)$ can then be used in Eq. (6) to obtain the coherent electric field pulse emitted by an arbitrary bunch shape.

\section{EXAMPLES}

In this section we present two examples that demonstrate the use of Eq. (19). The first shows that using cubic spline interpolation for a Gaussian bunch yields the same result obtained in Sec. II A. For the second example, we employ it to calculate the field of a complex bunch profile. This profile has been obtained by numerical simulations [17] of the linear accelerator FLUTE [3].

\section{A. Gauss vs spline}

Figure 2 shows a comparison of the electric field pulses of a Gaussian bunch calculated according to Eq. (9) and based on a spline interpolation and Eq. (19). The Gaussian profile was interpolated by cubic splines based on 9 equally spaced data points in the range from $-4 \sigma$ to $4 \sigma$. Other parameters were $Q=100 \mathrm{pC}, \sigma=50 \mathrm{fs}, \quad R=1 \mathrm{~m}$, $\rho=1 \mathrm{~m}$. The phase $\phi=45^{\circ}$ was chosen such that both real and imaginary parts of $\varepsilon(t)$ contribute equally to the electric field [see Eq. (6)]. Given that Eq. (9) was plotted with Mathematica whereas the spline interpolation and Eq. (19) were evaluated with a C++ program, the two answers agree very well.

\section{B. Simulated bunch profile at FLUTE}

FLUTE is a linear test accelerator for, amongst other purposes, the creation of intense THz pulses [3]. Currently under construction, it consists of an electron photoinjector, a linac, and a four dipole bunch compressor. A simulated longitudinal bunch profile of a $100 \mathrm{pC}$ bunch at the end of 


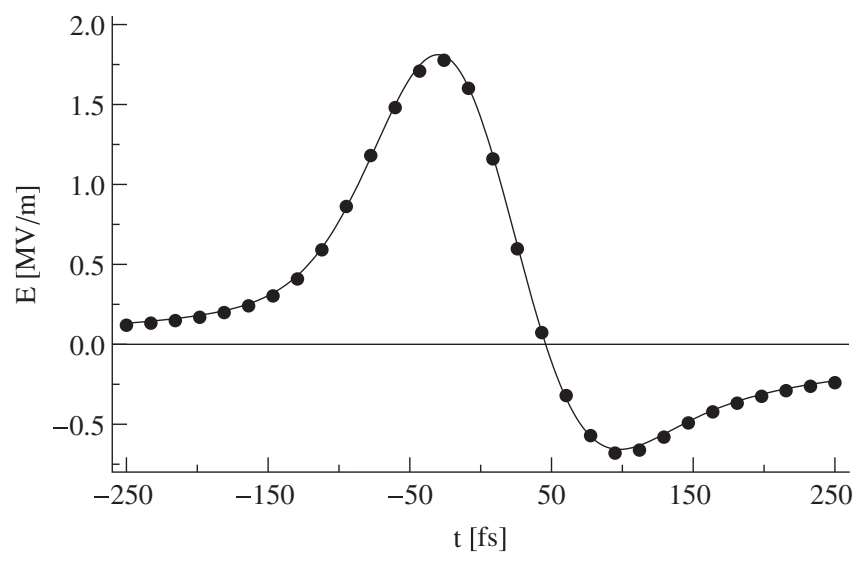

FIG. 2. Electric field of a $\mathrm{THz}$ pulse emitted by a $100 \mathrm{pC}$ Gaussian bunch of width $\sigma=50 \mathrm{fs}$ calculated according to Eq. (9) (continuous) and based on the cubic spline interpolation (points). The parameters were $R=1 \mathrm{~m}, \rho=1 \mathrm{~m}$, and $\phi=45^{\circ}$.

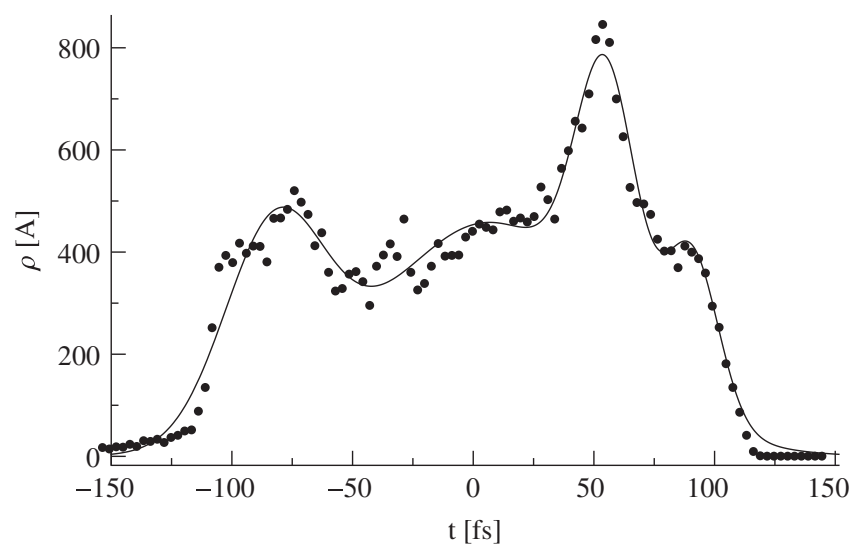

(a)

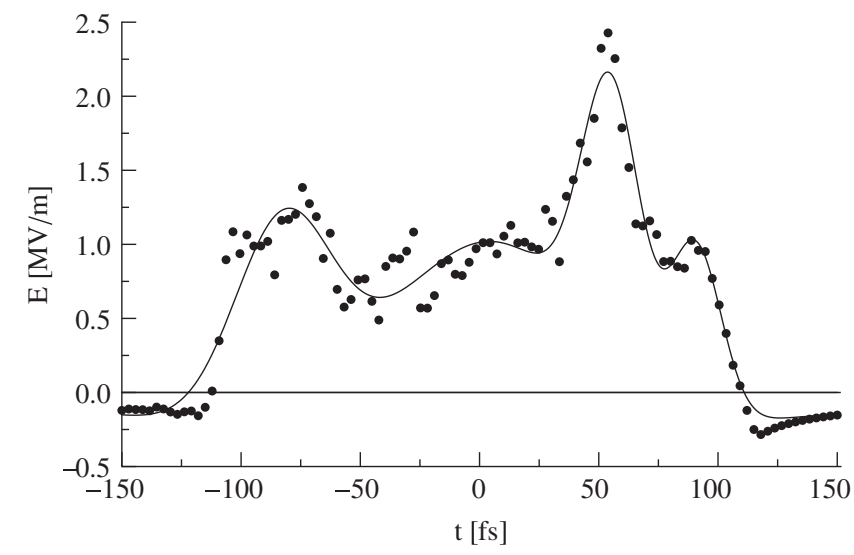

(b)

FIG. 3. (a) Simulated longitudinal charge profile of a $100 \mathrm{pC}$ bunch at FLUTE. Points are simulated data, while the continuous line is a fit of the data to a sum of four Gaussians. (b) Calculated electric field $E$ based on the multi-Gaussian fit and Eq. (9) (continuous) as well as the spline interpolation and Eq. (19) (points). Notice how, for the phase $\phi=0^{\circ}$ used here, each pulse shape follows its underlying temporal charge profile. the bunch compressor is shown in Fig. 3a [17], together with a fit of the profile to four Gaussians (continuous line). The simulations take into account the self-interaction of the bunch with its own radiation field in the compressor. This self-interaction gives rise to the complex substructure. Depending on the detector resolution of an experiment, the measured bunch profile will be more smooth. Here, we are mainly interested in benchmarking our methods for a complex bunch. The electric field pulse was calculated from this using [17] $\rho=1.108 \mathrm{~m}, R=1 \mathrm{~m}$, and $\phi=0^{\circ}$. Figure $3 \mathrm{~b}$ shows the electric field pulse using Eq. (9) for the multi-Gaussian fit (continuous curve) and Eq. (19) for the spline interpolation (points). Again, the two methods agree. Notice that the fields from the two methods closely follow their respective charge profiles for $\phi=0^{\circ}$. Since the multiGaussian fit does not "see" the substructure at around $-30 \mathrm{fs}$ in Fig. 3a, its electric field does not reproduce it in Fig. $3 b$ either. Similarly, since the multi-Gaussian fit correctly finds the position of the maximum current at about 53 fs but underestimates its magnitude by about $7 \%$,

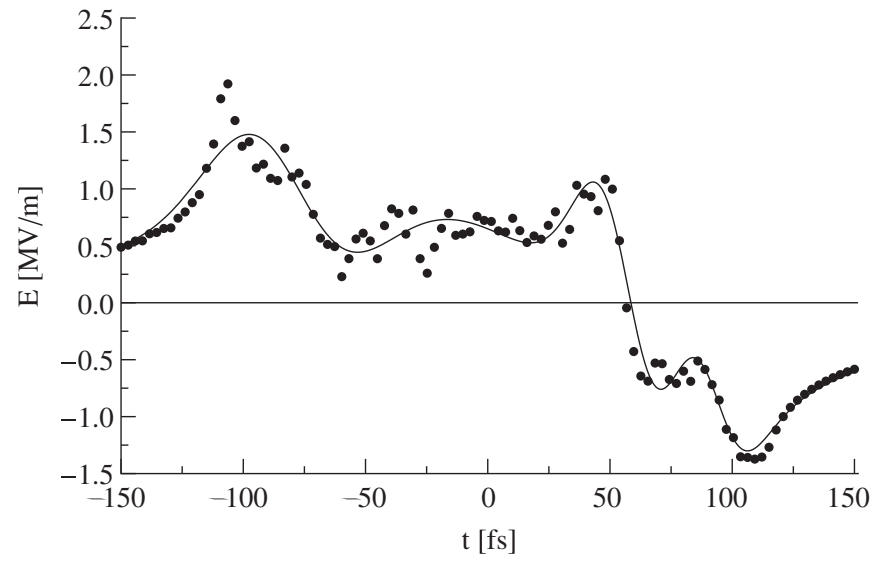

(a)

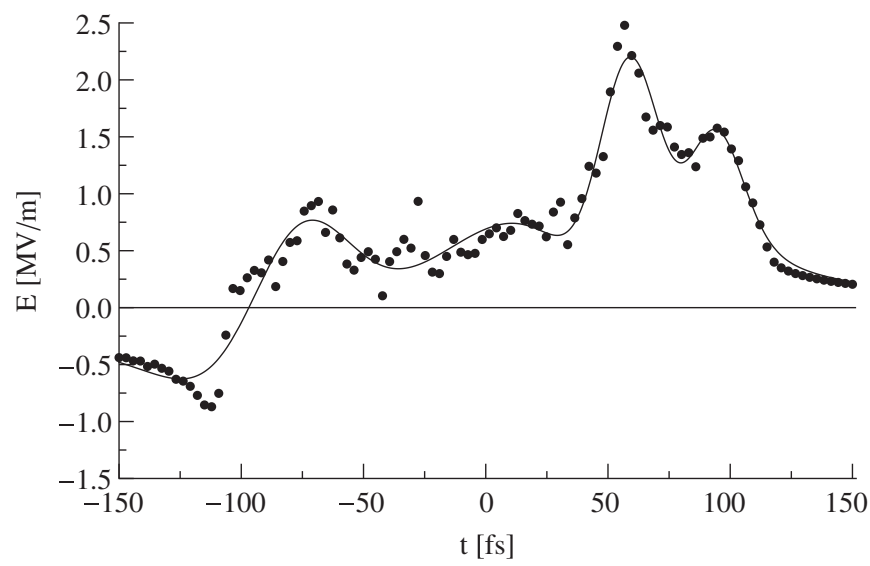

(b)

FIG. 4. Calculated electric field $E$ based on the multi-Gaussian fit and Eq. (9) (continuous) as well as the spline interpolation and Eq. (19) (points) for the longitudinal charge profile of Fig. 3a using $\phi=65^{\circ}$ (a) and $\phi=325^{\circ}$ (b). 
the resulting peak electric field at 53 fs is about $12 \%$ smaller.

Figure 4 shows the calculated electric field of the same bunch and parameters but for $\phi=65^{\circ}$ (Fig. 4a) and $\phi=325^{\circ}$ (Fig. 4b). As for the field of a simple Gaussian bunch shown in Fig. 1, minima develop at $t>0$ or $t<0$, respectively. Due to the complex substructure of the bunch, the peak field for $\phi \neq 0$ can still be as high as for $\phi=0$ (compare Fig. 3b with Fig. 4b).

\section{DISCUSSION}

Besides the cubic interpolation discussed here, it is possible to use a linear interpolation as well. It is obtained by setting $c_{3, i}=c_{2, i}=0$ in Eqs. (11). Provided the data points are sufficiently close, the two methods agree very well $[14,16]$.

The spectral amplitude $E_{0}(\omega)$ in Eq. (4) was obtained by integrating the spectral intensity over the entire solid angle of $4 \pi$. However, radiation of relativistic electrons is not isotropic but concentrated in a cone with an opening angle $\theta$ of the order of $1 / \gamma$. For the low-frequency synchrotron spectrum used here, we have [10]

$$
\theta=\frac{1}{\gamma}\left(\frac{2 \omega_{c}}{\omega}\right)^{1 / 3}
$$

With typical values for FLUTE [17], we have $\nu_{c} \simeq 36 \mathrm{THz}$ and frequencies around $1 \mathrm{THz}$ resulting in $\theta \simeq 4.2 / \gamma \simeq$ $51 \mathrm{mrad}$. At a distance of $1 \mathrm{~m}$ this gives a spot size radius of $51 \mathrm{~mm}$. Assuming focusing to the diffraction-limited spot radius given by $\lambda=0.3 \mathrm{~mm}$ then gives a focusing factor of about 170 by which the electric field strength is enhanced. For the unfocused pulse shown in Fig. 3b focusing means a peak field of about $425 \mathrm{MV} / \mathrm{m}$. The peak magnetic field is then $B_{\text {peak }}=E_{\text {peak }} / c \simeq 1.4 \mathrm{~T}$.

In this paper we did not take fully into account cutoff frequencies for the spectral integral in Eq. (3). A lower cutoff frequency is provided by shielding due to the vacuum chamber. As discussed in Sec. II, an upper cutoff frequency is provided either by the inverse bunch length $\omega_{b} \simeq 1 / \sigma_{b}$ or the critical frequency $\omega_{c}$ of the synchrotron spectrum. Our derivation holds for $\omega_{b} \ll \omega_{c}$. However, this condition can be violated for very short bunches. A treatment of this case is left for the future.

\section{ACKNOWLEDGMENTS}

The authors would like to thank M. Schmelling, as well as the ANKA THz group, for valuable discussions. M. S. acknowledges financial support by the Accelerator Research and Development program of the HelmholtzGemeinschaft.

[1] J. Feikes, K. Holldack, P. Kuske, and G. Wüstefeld, in Proceedings of the 9th European Particle Accelerator Conference, Lucerne, 2004 (EPS-AG, Lucerne, 2004), p. 2290 [http://accelconf.web.cern.ch/AccelConf/e04/].

[2] A.-S. Müller et al., in Proceedings of the 21st Particle Accelerator Conference, Knoxville, TN, 2005 (IEEE, Piscataway, NJ, 2005), p. 2518.

[3] M. J. Nasse et al., Rev. Sci. Instrum. 84, 022705 (2013).

[4] A.-S. Müller et al., Beam Dynamics Newsletter 57, 154 (2012).

[5] T. Qi, Y.-H. Shin, K.-L. Yeh, K. A. Nelson, and A. M. Rappe, Phys. Rev. Lett. 102, 247603 (2009).

[6] A.-S. Müller et al., in Proceedings of the 11th European Particle Accelerator Conference, Genoa, 2008 (EPS-AG, Genoa, Italy, 2008), p. 2094.

[7] A.-S. Müller, Rev. Accel. Sci. Techol. 03, 165 (2010).

[8] H. Wiedemann, Particle Accelerator Physics (Springer, New York, 2007), 3rd ed.

[9] R. Lai and A. Sievers, Nucl. Instrum. Methods Phys. Res., Sect. A 397, 221 (1997); G. Schneider, R. Lai, W. Walecki, and A. Sievers, Nucl. Instrum. Methods Phys. Res., Sect. A 396, 283 (1997).

[10] J. Jackson, Classical Electrodynamics (John Wiley \& Sons, New York, 1999), 3rd ed.

[11] M. Schwarz et al., in Proceedings of the 3rd International Particle Accelerator Conference, New Orleans, LA, 2012 (IEEE, Piscataway, NJ, 2012), p. 568.

[12] I. Gradshteyn and I. M. Ryzhik, Table of Integrals, Series, and Products (Elsevier, New York, 2007), 7th ed.

[13] K.-J. Kim, K. T. McDonald, G. V. Stupakov, and M. S. Zolotorev, Phys. Rev. Lett. 84, 3210 (2000).

[14] M. von Borstel, B.S. thesis, Karlsruhe Institute of Technology, 2013.

[15] W. H. Press et al., Numerical Recipes (Cambridge University Press, Cambridge, England, 2007), 3rd ed.

[16] P. Basler, B.S. thesis, Karlsruhe Institute of Technology, 2013.

[17] S. Naknaimueang et al., in Proceedings of the 34th International Free-Electron Laser Conference (Nara, 2012), p. 511. 